\title{
TNF Receptor Superfamily Members RANK, RANKL, OPG Pathway as Osteoimmunological Biomarker of Bone Healing after Orthognathic Surgery
}

\author{
İhsan O Osar ${ }^{1}$, Yavuz Fındık², Orhan Akpinar ${ }^{3}$
}

\begin{abstract}
Tumor necrosis factor (TNF) receptor superfamily member receptor activator of nuclear factor-KB (RANK), RANK-ligand (RANKL), and osteoprotegerin (OPG) pathway as osteoimmunological biomarker of bone healing after surgery. These biomarkers play an important role in the bone repair and healing after orthognathic surgery. The aim of our study was to evaluate levels of the RANKL-RANK-OPG cytokine system as osteoimmunological biomarker of bone healing after orthognathic surgery. Receptor activator of nuclear factor-KB, RANKL, and OPG were evaluated in 25 patients who is undergoing orthognathic surgery. Blood samples were collected before the operation, after 1 st day, and 10 th day of the operation. Differences among OPG, RANK, and RANKL averages were not statistically significant in all time levels and operation types. In this study, we concluded that RANKL/OPG ratio and blood serum RANK levels may show bone remodeling activity and bone remodeling activity after orthognathic surgery, although there is a need for further extensive studies.
\end{abstract}

Keywords: Biomarker, General anesthesia, Orthognathic surgery.

International Journal of Experimental Dental Science (2020): 10.5005/jp-journals-10029-1201

\section{INTRODUCTION}

Orthognathic surgery is the main procedure for the correction of jaw function and esthetics of the face. Orthognathic surgery is performed on the surgeon's bone, but the processes on the bone activate the bone formation and destruction events on this tissue. During the healing period, many bone-related mediators can play an important role and levels of these mediators change after the orthognathic surgery. ${ }^{1}$ Osteoblast and osteoclast interactions have an important role in skeletal homeostasis. ${ }^{2}$ This interaction is seriously supervised by different systems, such as the endocrine system, and is influenced by the immune system, an osteoimmunological regulation. ${ }^{2,3}$ The role of three novel members of the tumor necrosis factor ligand and receptor superfamily, the receptor activator of nuclear factor-KB (RANK), its ligand (RANK-ligand or RANKL), and the soluble decoy receptor of RANKL named osteoprotegerin (OPG) have assisted in a significantly to the establishment of osteoimmunology. ${ }^{2-4}$ Osteoprotegerin/ RANK/RANKL affected bone system, immune system, and vascular systems. Osteoprotegerin, RANK, and RANKL secrete selectively by numerous cell types and a variety of tissues, such as lymphocytes and osteoblasts. ${ }^{4}$ RANK-ligand intensely induces osteoclastogenesis and then bone resorption via its binding to RANK. Osteoprotegerin avoids osteolysis and impedes the RANKL/RANK interaction. Although the OPG/RANK/RANKL pathway is the main actor of bone apposition/resorption system, other controls, such as TNF-a and IL-1, exists. ${ }^{2,5}$ The overall data explain that OPG/RANK/RANKL comprises a molecular bridge to the bone homeostasis and immunity called osteoimmunology. ${ }^{5}$ These cytokine system is one of the most important mediators in the maintenance of bone cell function and in the activation of bone remodeling. The interaction between osteoblasts and osteoclasts has immunopathological \begin{tabular}{l}
\hline 1-3 Department of Oral and Maxillofacial Surgery, Faculty of Dentistry, \\
Süleyman Demirel University, Isparta, Turkey \\
Corresponding Author: Yavuz Fındık, Department of Oral and \\
Maxillofacial Surgery, Faculty of Dentistry, Süleyman Demirel University, \\
Isparta, Turkey, Phone: +902462113251, e-mail: yavuzfindik32@ \\
hotmail.com \\
How to cite this article: Osar iO, Fındık Y, Akpinar O, et al. TNF \\
Receptor Superfamily Members RANK, RANKL, OPG Pathway as \\
Osteoimmunological Biomarker of Bone Healing after Orthognathic \\
Surgery. Int J Experiment Dent Sci 2020;9(1):4-7. \\
Source of support: Süleyman Demirel University \\
Conflict of interest: Nil \\
\hline \hline
\end{tabular}

implications associated with either a decrease or increase of bone mass mineral density.

The aim of our study was to assess the levels of the RANKLRANK-OPG cytokine system after the orthognathic surgery.

\section{Materials and Methods \\ Ethics}

Patient consent form, and study design was approved by the ethics committees. The study was approved by the human ethics committees of the Faculty of Medicine of Süleyman Demirel University, Isparta, Turkey (05.04.2017-no:54). Informed consent was obtained from the patients before the surgery.

\section{Patients}

Our study was carried out on 25 individuals in our Department of Oral and Maxillofacial Surgery, Faculty of Dentistry, Süleyman

(0) The Author(s). 2020 Open Access This article is distributed under the terms of the Creative Commons Attribution 4.0 International License (https://creativecommons. org/licenses/by-nc/4.0/), which permits unrestricted use, distribution, and non-commercial reproduction in any medium, provided you give appropriate credit to the original author(s) and the source, provide a link to the Creative Commons license, and indicate if changes were made. The Creative Commons Public Domain Dedication waiver (http://creativecommons.org/publicdomain/zero/1.0/) applies to the data made available in this article, unless otherwise stated. 
Demirel University, 2017, who were in need of orthognathic surgery in accordance with our patient selection criteria.

The following additional criteria were met by all participants:

- The decision of the patient to be made by the joint decision of the orthodontist and jaw surgeons with the indication of orthognathic surgery.

- No systemic disturbance that may adversely affect growth and development.

- No genetic or congenital craniofacial deformity.

- Good oral hygiene.

All patients were healthy without any systemic or bone diseases. Orthognathic surgery was applied to all patients.

\section{Surgical Technique}

Orthognathic surgery (both maxillary operations and mandibular operations) has been selected as a model surgery in our study. All patients were informed about the surgical procedure and the schedule of blood samples and an informed consent was assigned. Orthognathic surgery was applied to 15 females and 10 males. In 13 patients Le Fort I osteotomy and in 12 patients bilateral sagittal split ramus osteotomy (BSSRO) was performed. Nasoendotracheal intubation was performed and after completing the osteotomies according to the techniques bone fixations were provided in the normal occlusion with the help of splints. Osteotomy lines were rigidly fixed with $2.0 \mathrm{~mm}$ titanium plate and screws.

\section{Blood Sample}

For determining the values of RANK, RANKL, and OPG in the blood be taken from the blood sample (control group) before the operation and the blood sample (study group) again after 1st day and 10th day of the operation. Blood samples were centrifuged for 5 minutes at 4,000 rpm in 30 minutes and their sera were separated. Serum was taken into $2 \mathrm{~mL}$ Eppendorf tubes and stored in the freezer at $-80^{\circ} \mathrm{C}$ until the working day.

\section{ELISA Tests}

It is aimed to determine the amounts of RANK, RANKL, and OPG in serum samples in these tubes with the help of ELISA devices. Analyses of osteoimmunological biomarkers were performed in the Faculty of Dentistry Research Laboratory. Analysis of the levels of RANK used the Human RANK PicoKine ${ }^{\text {TM }}$ ELISA Kit with the Catalog Number of EK0829, purchased from Boster Biological Technology (Pleasanton, USA). Analysis of the levels of RANKL used the "Human RANKL PicoKine ${ }^{\mathrm{TM}}$ ELISA Kit" with the Catalog Number EK0842, purchased from Boster Biological Technology (Pleasanton, USA). Analysis of the levels of OPG used the "Human OPG PicoKine ${ }^{\mathrm{TM}}$ ELISA Kit" with the Catalog Number EK0480 purchased from Boster Biological Technology (Pleasanton, USA). The analysis was performed according to the detailed procedures set forth in the kit.

\section{Statistical Analysis}

For analyzing the statistical values, we used "SPSS (Statistical Package for Social Sciences) for Windows 15.0 program". The data obtained for the characteristics studied in the study were the Kolmogorov-Smirnov test and the homogeneity of the normal distribution and variance-covariance items, which are prerequisites of the parametric tests. It has been found that the prerequisites cannot be achieved by looking at boxing " $M$ " test. Therefore, the Freedman test was used to compare the time of each operation method, and the Kruskal-Wallis test was used to compare the operation types at each time. Mann-Whitney $\mathrm{U}$ test was used for gender $(p<0.05)$.

\section{Results}

Fifteen females and 10 males (mean age 22.3) was included in this study. Differences among OPG, RANK, and RANKL averages as a result of OPG, RANK, and RANKL tests were not statistically significant in patients who had undergone Le Fort 1 operation, preoperatively, on the 1st day of operation and on the 10th day of surgery (Table 1).

Differences among OPG, RANK, and RANKL averages were not statistically significant as a result of the Friedman test in terms of OPG, RANK, and RANKL values in the patients who underwent BSSRO before surgery, on the 1st day of surgery, and on the 10th day of surgery (Table 2).

Mann-Whitney $U$ test was used to determine the OPG, RANK, and RANKL values of serum samples taken before surgery, day 1 , and day 10 of surgery, and the mean of the sexes was not statistically significant.

\section{Discussion}

Hideous activation of the TNF superfamily of cytokines induces myriad pathological conditions. ${ }^{2}$ But biological agents have had positive effects on the course of these diseases, each agent carries substantial complications. ${ }^{3}$ According to the recent research, a number of cytokines have been identified in the TNF family in the control of bone formation-destruction. These molecules are RANKL, RANK, and OPG. These three molecules affect among bone, immune cells, and vascular system. Therefore, they give new strategies for the treatment and protection of diseases related to them. Since OPG/RANKL/RANK pathway interacts between bone metabolism and immune response, it has been evaluated under the title of osteoimmunology. ${ }^{2-4}$ Apart from cell migration, there are data showing that OPG/RANK/RANKL system is involved in the inflammatory process. Numerous cytokines are involved as regulators in bone response to inflammation. ${ }^{2,4}$

This study was the first in terms of the investigation of the relationship between OPG/RANKL/RANK values and bone formation degradation in patients undergoing Le Fort 1 surgery and bilateral sagittal split osteotomy in vivo. In our study, there was no significant difference in terms of RANKL, OPG level in patients with operation techniques, gender differences, and timing of serum samples taken.

Several studies were performed in the literature for evaluating these biomarkers in different operations. ${ }^{6-9}$ In a study, effect of bone healing on osteoprotegerin was investigated after orthognathic surgery. They have planned OPG evaluation for a period of 6 weeks after jaw surgery. Osteoprotegerin was measured for 12 weeks and concluded that the variation was significant at 4 weeks postoperatively and not significant at 8 and 12 weeks postoperatively. ${ }^{10}$ In another study, researchers showed that serum OPG value was increased after the surgical procedure. ${ }^{11}$ Colombini et al. studied the role of OPG in the healing of humerus fractures at different time points before and after the surgery and they stated that these values did not show any significant difference. ${ }^{12}$ In an animal study, Arnez et al. evaluated osteoclastogenesis signaling in midpalatal suture after rapid maxillary expansion (RME) in rats. Different from our results, they concluded that there was a serious secretion of RANK and RANKL 
Table 1: Results of the Le Fort I surgery

\begin{tabular}{|c|c|c|c|c|c|c|}
\hline \multicolumn{7}{|l|}{ Le Fort 1 surgery } \\
\hline Blood sample & $n$ & Mean & Std. deviation & Minimum & Maximum & $p$ value \\
\hline \multicolumn{7}{|l|}{ OPG } \\
\hline Preoperatively & 13 & 58.85 & 20.66 & 40.63 & 95.09 & 0.12 \\
\hline Postop. 1st day & 13 & 60.26 & 22.90 & 38.86 & 96.41 & 0.17 \\
\hline Postop. 10th day & 13 & 75.49 & 79.55 & 23.75 & 235.28 & 0.34 \\
\hline \multicolumn{7}{|l|}{ RANK } \\
\hline Preoperatively & 13 & 48.72 & 2.79 & 44.36 & 52.22 & 0.66 \\
\hline Postop. 1st day & 13 & 49.72 & 5.14 & 45.30 & 58.93 & 0.45 \\
\hline Postop. 10th day & 13 & 51.90 & 4.16 & 46.59 & 57.30 & 0.78 \\
\hline \multicolumn{7}{|l|}{ RANKL } \\
\hline Preoperatively & 13 & 10.83 & 14.29 & 0.02 & 38.17 & 0.23 \\
\hline Postop. 1st day & 13 & 9.59 & 7.45 & 1.45 & 20.24 & 0.67 \\
\hline Postop. 10th day & 13 & 13.85 & 15.87 & 0.60 & 43.27 & 0.34 \\
\hline
\end{tabular}

Table 2: Results of the BSSRO

\begin{tabular}{|c|c|c|c|c|c|c|}
\hline \multicolumn{7}{|l|}{ BSSRO } \\
\hline Blood sample & $n$ & Mean & Std. deviation & Minimum & Maximum & $p$ value \\
\hline \multicolumn{7}{|l|}{ OPG } \\
\hline Preoperatively & 12 & 55.03 & 35.67 & 12.05 & 114.59 & 0.85 \\
\hline Postop. 1st day & 12 & 81.29 & 26.22 & 51.44 & 132.35 & 0.67 \\
\hline Postop. 10th day & 12 & 67.75 & 27.81 & 42.44 & 113.65 & 0.60 \\
\hline \multicolumn{7}{|l|}{ RANK } \\
\hline Preoperatively & 12 & 62.73 & 34.02 & 47.92 & 146.27 & 0.57 \\
\hline Postop. 1st day & 12 & 60.49 & 30.73 & 46.51 & 136.21 & 0.81 \\
\hline Postop. 10th day & 12 & 56.89 & 14.56 & 45.70 & 90.92 & 0.41 \\
\hline \multicolumn{7}{|l|}{ RANKL } \\
\hline Preoperatively & 12 & 395.85 & 1042.71 & 6.34 & 2975.38 & 0.93 \\
\hline Postop. 1st day & 12 & 288.83 & 755.50 & 0.02 & 2157.29 & 0.97 \\
\hline Postop. 10th day & 12 & 132.78 & 250.27 & 5.73 & 740.34 & 0.90 \\
\hline
\end{tabular}

genes at 7 and 10 days and an upregulation of the OPG gene at 3 and 7 days of healing. ${ }^{13}$

In our conclusion, the blood serum RANK, RANKL, and OPG ratios at the time of presentation were variable at a certain rate in any of the treatments performed on the bone and in previous bone disease studies. The discrepancy between the various studies may be explained by reasons such as the OPG/RANKL system change will not be specific to bone metabolism and the sensitivity and orderliness of existing ELISA tests due to the synthesis of OPG, RANK, and RANKL beside the bone in other tissues. With the addition of osteoimmunological information, the pathogenesis of bone resorption in orthognathic surgery and the underlying mechanisms of remodeling and many inflammatory diseases will begin to be better understood. The ratio of RANKL/OPG and blood serum RANK levels may be diagnostic for the rate of recovery in orthognathic surgery. In this study, we concluded that RANKL/OPG ratio and blood serum RANK levels may show bone remodeling activity and bone remodeling activity after orthognathic surgery, although there is a need for further extensive studies. Using the OPG fusion protein and other RANK-mediated osteoclastogenesis inhibitors, modulation of the RANKL-RANK-OPG axis may be possible in the near future.

\section{References}

1. Broers DLM, van der Heijden GJMG, Rozema FR, et al. Do patients benefit from orthognathic surgery? A systematic review on the effects of elective orthognathic surgery on psychosocial functioning and patient satisfaction. Eur J Oral Sci 2017;125(6):411-418. DOI: 10.1111/ eos.12371.

2. Takayanagı Hiroshi. Osteoimmunology and the effects of the immune system on bone. Nat Rev Rheumatol 2009;5((12):667. DOI: 10.1038/ nrrheum.2009.217.

3. Walsh MC, Kim N, Kadono Y, etal. Osteoimmunology:interplay between the immune system and bone metabolism. Annu Rev Immunol 2006;24(1):33-63. DOI: 10.1146/annurev.immunol.24.021605.090646.

4. Theoleyre S, Wittrant Y, Tat SK, et al. The molecular triad OPG/RANK/ RANKL: involvement in the orchestration of pathophysiological bone remodeling. Cytok Grow Fact Rev 2004;15(6):457-475. DOI: 10.1016/j. cytogfr.2004.06.004.

5. Walsh MC, Choi Y. Biology of the TRANCE axis. Cytokine Growth Factor Rev 2003;14(3-4):251-263. DOI: 10.1016/s1359-6101(03)00027-3.

6. Mehrara BJ, Rowe NM, Steinbrech DS, et al. Rat mandibular distraction osteogenesis: II. Molecular analysis of transforming growth factor beta-1 and osteocalcin gene expression. Plast Reconstr Surg 1999;103(2):536-547. DOI: 10.1097/00006534-199902000-00026.

7. Bostanci N, llgenli T, Emingil G, et al. Gingival crevicular fluid levels of RANKL and OPG in periodontal diseases: implications of their relative 
ratio. J Clin Periodontol 2007;34(5):370-376. DOI: 10.1111/j.1600051X.2007.01061.x.

8. Mogi M, Otogoto J, Ota N, et al. Differential expression of RANKL and osteoprotegerin in gingival crevicular fluid of patients with periodontitis. J Dent Res 2004;83(2):166-169. DOI: 10.1177/154405910408300216.

9. Ogasawara T, Yoshimine Y, Kiyoshima T, et al. In situ expression of RANKL, RANK, osteoprotegerin, and cytokines in osteoclasts of rat periodontal tissue. J Periodont Res 2004;39(1):42. DOI: 10.1111/j.16000765.2004.00699.x.

10. Soliman S, Ahmed M. The effect of orthognathic surgery on osteoprotegerin as immunological caliper of bone healing. Open Access Maced J Med Sci 2016;4(4):705-708. DOI: 10.3889/ oamjms.2016.123.
11. Lee JS, Ryu CH, Moon NH, et al. Changes in serum levels of receptor activator of nuclear factor-kB ligand, osteoprotegerin, IL-6 and TNF in patients with a concomitant head injury and fracture. Arch Orthop Trauma Surg 2009;129(5):711-718. DOI: 10.1007/s00402-0080632-8.

12. Colombini A, Lombardi G, Galliera E, et al. Plasma and drainage fluid levels of soluble receptor activator of nuclear factor-kB (sRANK), soluble receptor activator of nuclear factor-kB ligand (sRANKL) and osteoprotegerin (OPG) during proximal humerus fracture healing. Int Orthop 2011;35(5):777-782. DOI: 10.1007/s00264-0101088-3.

13. Arnez MFM, Ribeiro LSN, Barretto GD, et al. RANK/RANKL/OPG expression in rapid maxillary expansion. Braz Dent J 2017;28(3): 296-300. DOI: 10.1590/0103-6440201601116. 